\title{
Ampliação da distribuição de quatro espécies de morcegos (Mammalia, Chiroptera) no Rio Grande do Sul, Brasil
}

\author{
Marcelo de Moraes Weber ${ }^{1,2}$, Jeferson Luis Steindorff de Arruda ${ }^{1}$ \& Nilton Carlos Cáceres ${ }^{1}$ \\ Biota Neotropica $v 7(n 2)$ \\ http://www.biotaneotropica.org.br/v7n2/pt/abstract?short-communication+bn01307022007
}

\author{
Recebido em 01/08/06 \\ Versão Reformulada recebida em 15/01/07 \\ Publicado em 01/05/07
}
${ }^{1}$ Laboratório de Ecologia de Mamíferos, Departamento de Biologia, Universidade Federal de Santa Maria, Avenida Roraima, 1000, CEP 97015-900, Santa Maria, RS, Brasil
${ }^{2}$ Autor para correspondência: Marcelo de Moraes Weber, e-mail:marcelo_weber@yahoo.com.br,www.ufsm.br

\begin{abstract}
Weber, M.M., Arruda, J.L.S. \& Cáceres, N.C. Extension of the distribution of four bat species (Mammalia, Chiroptera) in Rio Grande do Sul, Brazil. Biota Neotrop. May/Aug 2007 vol. 7, no. 2 http://www.biotaneotropica. org.br/v7n2/pt/abstract?short-communication+bn01307022007. ISSN 1676-0603.

The objective of this paper is to record the occurrence of four bats species in the central of the Rio Grande do Sul State, south of Brazil: Histiotus montanus, Pygoderma bilabiatum, Nyctinomops laticaudatus and Myotis ruber. Data were obtained from the scientific collection of Universidade Federal de Santa Maria (UFSM) and represent distribution extensions for these bat species in this State. We present information on the habitat where the species were collected, showing that some of them are new for this species. Some of the studied species may be considered rare in the Rio Grande do Sul State, whereas others should have their degree of threat revaluated.

Keywords: distribution, Histiotus montanus, Myotis ruber, Nyctinomops laticaudatus, Pygoderma bilabiatum.

\section{Resumo}

Weber, M.M., Arruda, J.L.S. \& Cáceres, N.C. Ampliação da distribuição de quatro espécies de morcegos (Mammalia, Chiroptera) no Rio Grande do Sul, Brasil. Biota Neotrop. May/Aug 2007 vol. 7, no. 2 http://www. biotaneotropica.org.br/v7n2/pt/abstract?short-communication+bn01307022007. ISSN 1676-0603.

Este estudo tem por objetivo registrar a ocorrência de quatro espécies de morcegos na região central do Estado do Rio Grande do Sul, sul do Brasil: Histiotus montanus, Pygoderma bilabiatum, Nyctinomops laticaudatus e Myotis ruber. Os dados foram obtidos a partir da coleção científica da Universidade Federal de Santa Maria (UFSM) e representam ampliações de distribuição para essas espécies no Estado. São fornecidas informações sobre o habitat onde as espécies foram coletadas, sendo que alguns desses são novos para essas espécies. Pode-se considerar que algumas das espécies enfocadas são raras no Rio Grande do Sul, ao passo que outras deveriam ter o seu grau de ameaça reconsiderado.
\end{abstract}

Palavras-chave: distribuição, Histiotus montanus, Myotis ruber, Nyctinomops laticaudatus, Pygoderma bilabiatum. 


\section{Introdução}

No Rio Grande do Sul são registradas 36 espécies de morcegos (Weber et al. 2006), pertencentes a quatro famílias: Molossidae, Noctilionidae, Phyllostomidae e Vespertilionidae (Pacheco \& Marques 2006). Elas se distribuem nos biomas Campos Sulinos e Mata Atlântica, os quais são típicos desse Estado. A maioria das espécies habita ambientes florestais (Marinho-Filho \& Sazima 1998), embora existam espécies muito bem adaptadas ao ambiente urbano (Fabián et al. 1990, Sazima et al. 1994).

São poucos os trabalhos que tratam da distribuição geográfica dos morcegos no Rio Grande do Sul, ressaltando-se os estudos de Voss (1973), Silva (1975), Silva \& Souza (1980), Wallauer \& Albuquerque (1986), González \& Fabián (1995), Fabián et al. (1999), Rui et al. (1999) e Fabián et al. (2006). A maioria desses estudos retrata principalmente quirópteros presentes em Unidades de Conservação, localizadas nos extremos norte e leste do Estado, ficando as regiões central, oeste e sul carentes de informações básicas sobre a distribuição e ecologia desse grupo.

O objetivo deste estudo é registrar as novas ocorrências dos morcegos Histiotus montanus (Philippi \& Landbeck, 1861) e Myotis ruber (E. Geoffroy, 1806) (Vespertilionidae), Nyctinomops laticaudatus (E. Geoffroy, 1805) (Molossidae) e Pygoderma bilabiatum (Wagner, 1843) (Phyllostomidae, Stenoderminae) na região central do Estado do Rio Grande do Sul, ampliando, dessa forma, suas respectivas áreas de distribuição.

\section{Material e Métodos}

O Rio Grande do Sul se localiza na transição das zonas climáticas tropical e subtropical, o que explica, em parte, a formação de campos e de florestas nesse Estado (Marchiori 2004). A região central, localizada na área de transição entre o Planalto Rio-Grandense e a Depressão Central, possui altitude máxima em torno de $460 \mathrm{~m}$ e sua vegetação é caracterizada, por campos nas planícies e florestas estacionais nas encostas.

Os dados apresentados nessa comunicação foram obtidos por meio da análise dos morcegos tombados na coleção científica da Universidade Federal de Santa Maria (UFSM) e complementados com dados compilados da literatura. São apresentados também dados referentes aos métodos, horários e locais de coleta retirados das fichas de campo dos espécimes analisados.

\section{Resultados e Discussão}

Histiotus montanus distribui-se na América do Sul, ao longo da Cordilheira dos Andes, incluindo Venezuela, Colômbia, Equador, Peru, Bolívia, Chile, Argentina, Uruguai e sul do Brasil (Koopman 1982). No Brasil, essa espécie é somente registrada para o Rio Grande do Sul (Voss 1973, Fabián et al. 2006), Santa Catarina (Wallauer et al. 2000, Cherem et al. 2004) e Paraná (Miranda et al. 2006). No Rio Grande do Sul, H. montanus possui registros para o extremo leste, em área de Floresta Ombrófila Mista, nos municípios de São Francisco de Paula $\left(29^{\circ} 24^{\prime} \mathrm{S}\right.$ e $\left.50^{\circ} 22^{\prime} \mathrm{W}\right)$, Maquiné (29 $39^{\prime} \mathrm{S}$ e $50^{\circ} 12^{\prime} \mathrm{W}$ ) (S.M. Pacheco, com. pes.) e Ilópolis ( $28^{\circ} 55^{\prime} \mathrm{S}$ e $52^{\circ} 07^{\prime} \mathrm{W}$ ) (Fabián et al. 2006). Recentemente foi confirmada a ocorrência dessa espécie nos municípios de Bagé ( $\left.31^{\circ} 21^{\prime} \mathrm{S}, 54^{\circ} 03^{\prime} \mathrm{W}\right)$ e Pinheiro Machado ( $31^{\circ} 34^{\prime} \mathrm{S}, 5^{\circ} 22^{\prime}$ W) em formações estépicas (Fabián et al. 2006) (Figura 1a).

Foi examinado um indivíduo macho de $H$. montanus (ZUFSM 0341) coletado durante a noite de 13/05/05 às 21:40 horas (3 horas e 20 minutos após o pôr-do-sol) no município de Santa Maria no "Morro do Elefante" (29 40' S e 53 43' W, $262 \mathrm{~m}$ de altitude), com a utilização de redes de neblina em área de Floresta Estacional
Decidual. O exemplar apresentava antebraço de 45,2 mm, corpo de $52,0 \mathrm{~mm}$, massa de 10,4 g e não apresentava testículos evidentes. A ocorrência dessa espécie em Santa Maria representa uma expansão de sua área de distribuição e a ocupação de uma nova formação vegetal não registrada anteriormente no Rio Grande do Sul, não sendo essa, uma espécie abundante no Estado, conforme sugerido por Fabián et al. (2006).

Pygoderma bilabiatum tem distribuição disjunta na América do Sul, ocorrendo no Suriname, Bolívia, Paraguai, norte da Argentina (Misiones) (Koopman 1982) e regiões centro-oeste, sudeste e sul do Brasil (Peracchi et al. 2006). Para a Argentina, Barquez et al. (1993) citam esta espécie como rara no noroeste e como comum no nordeste desse país. No Rio Grande do Sul, P. bilabiatum possui registros somente para o nordeste e extremos norte e noroeste do Estado, nos municípios de Derrubadas ( $27^{\circ} 10^{\prime} \mathrm{S}$ e $53^{\circ} 75^{\prime} \mathrm{W}$ ),

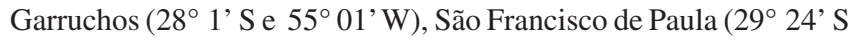
e $50^{\circ} 22^{\prime} \mathrm{W}$ ) e Maquiné (29 $\left.39^{\prime} \mathrm{S}, 50^{\circ} 12^{\prime} \mathrm{W}\right)$, ocupando áreas de Floresta Estacional Decidual, Floresta Ombrófila Densa e Mista (Fabián et al. 1999) (Figura 1b).

Foram examinados dois indivíduos machos de $P$. bilabiatum (ZUFSM 0410 e ZUFSM 0345). O primeiro indivíduo foi coletado no dia 29/08/1998 no município de Ibarama (29² $24^{\prime}$ S e $\left.53^{\circ} 13^{\prime} \mathrm{W}\right)$ com a utilização de redes-de-neblina e apresentava antebraço de $38,5 \mathrm{~mm}$ e corpo de $48,5 \mathrm{~mm}$. O segundo exemplar analisado (ZUFSM 0345) foi capturado no município de Santa Maria no "Morro do Elefante" (29 40' S, 53 43' W, 262 m de altitude), no dia 09/07/05 às 05:00 horas, com a utilização de redes-de-neblina e apresentava antebraço de 37,4 mm, corpo de 52,0 mm e massa de 19,0 g. Ambos não apresentavam testículos evidentes.

A ocorrência desta espécie em área de Floresta Estacional Decidual nos municípios de Santa Maria e Ibarama representa uma interiorização da mesma no Rio Grande do Sul, já que era somente registrada para os limites extremos do Estado (Fabian et al. 1999). Ainda se conhece muito pouco sobre a biologia dessa espécie e, portanto, estudos em regiões com formações vegetais diferentes são aconselháveis para uma melhor compreensão da distribuição e ecologia dessa espécie no Estado do Rio Grande do Sul.

Nyctinomops laticaudatus apresenta ampla distribuição na América do Sul, ocorrendo desde a Guiana Francesa e Colômbia até Argentina e Brasil (Koopman 1982). Nyctinomops laticaudatus consta no Livro Vermelho da Fauna Ameaçada de Extinção do Rio Grande do Sul e possui registros somente para três localidades do Estado: Estação Ecológica do Taim, nos municípios de Rio Grande (32 $03^{\circ} \mathrm{S}$

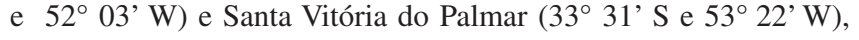
Porto Alegre ( $30^{\circ} 03^{\prime} \mathrm{S}$ e $\left.51^{\circ} 11^{\prime} \mathrm{W}\right)$ (Pacheco \& Freitas 2003) e Torres (29 $18^{\prime}$ S e $\left.49^{\circ} 41^{\prime} \mathrm{W}\right)$ (Silva \& Souza 1980) (Figura 1c). Essas localidades apresentam características florísticas diferentes. $\mathrm{O}$ município de Torres (29 $18^{\prime} \mathrm{S}$ e $\left.49^{\circ} 41^{\prime} \mathrm{W}\right)$ está localizado em área de Floresta Ombrófila Densa (Floresta Atlântica), enquanto que Porto Alegre $\left(30^{\circ} 03^{\prime} \mathrm{S}\right.$ e $\left.51^{\circ} 11^{\prime} \mathrm{W}\right)$ está localizado em área de Floresta Estacional Semidecidual e Rio Grande ( $32^{\circ} 03^{\prime} \mathrm{S}$ e $52^{\circ} 03^{\prime} \mathrm{W}$ ) e

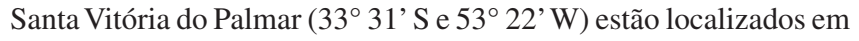
área de Restinga (Quadros \& Pillar 2002). A Estação Ecológica do Taim situa-se na faixa litorânea e é constituída por lagoas costeiras associadas a banhados, matas paludosas e campos. Em Torres, essa espécie foi encontrada nas Furnas da Guarita (29 $1^{\circ}$ 'S e $49^{\circ} 44^{\prime}$ W) presa à rocha basáltica à beira do Oceano Atlântico (Silva \& Souza 1980).

Foram examinados três exemplares (ZUFSM 0340, ZUFSM 0411, ZUFSM 0412) de N. laticaudatus, todos coletados no campus da Universidade Federal de Santa Maria (UFSM) (29 40' S e $53^{\circ} 43^{\prime} \mathrm{W}$ ) através de captura manual. Esta espécie utiliza como abrigos diurnos frestas existentes nos prédios dessa instituição e aparenta 


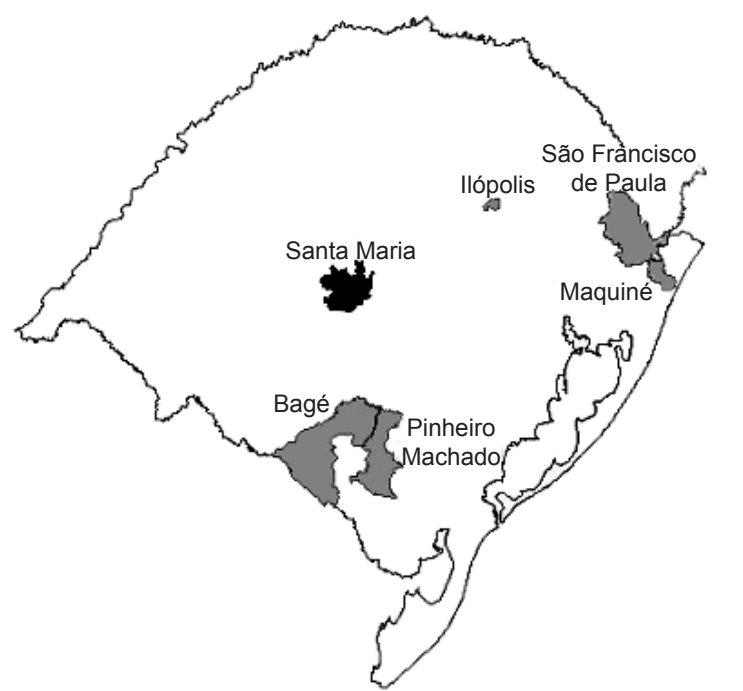

(a)

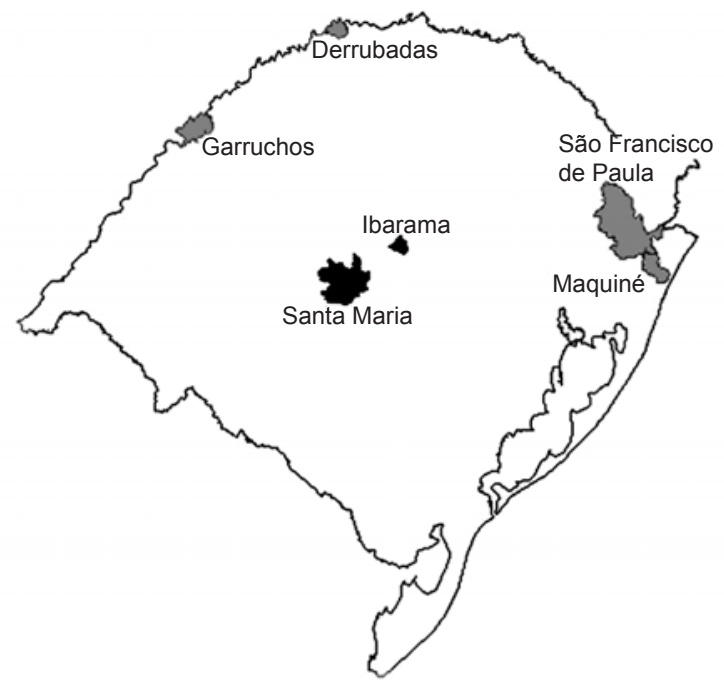

(b)

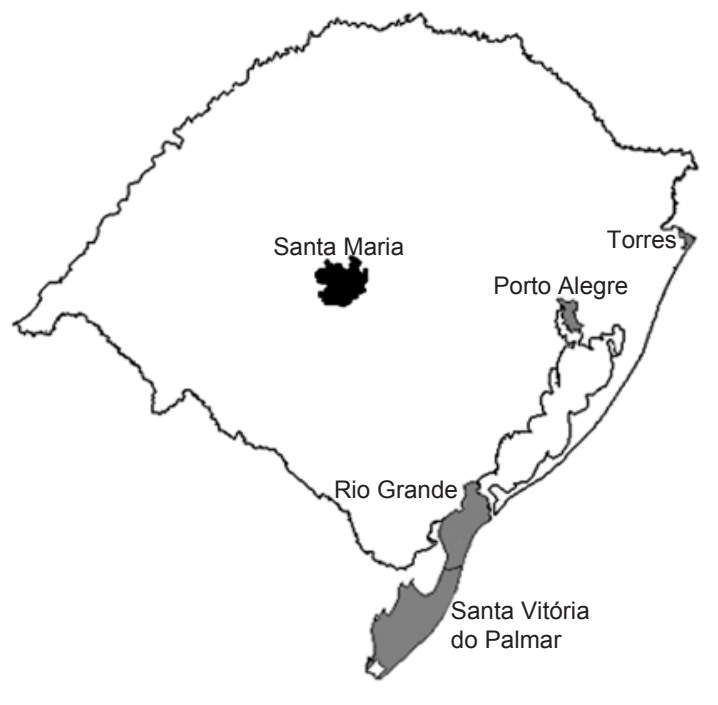

(C)

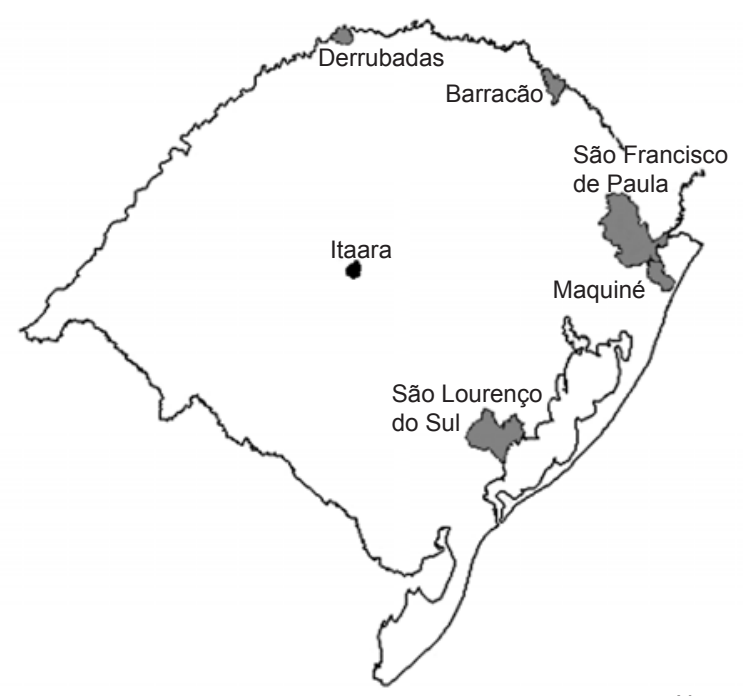

(d)

Novo registro $\square$ Distribuição conhecida

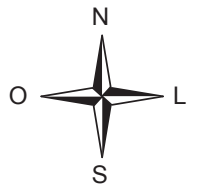

Figura 1. Distribuição de quatro espécies de morcegos no Estado do Rio Grande do Sul, Brasil. a) Histiotus montanus; b) Pygoderma bilabiatum; c) Nyctinomops laticaudatus; e d) Myotis ruber.

Figure 1. Distribution of four bat species in the Rio Grande do Sul State. a) Histiotus montanus; b) Pygoderma bilabiatum; c) Nyctinomops laticaudatus; and d) Myotis ruber.

ser abundante no campus da UFSM, sugerindo que novos estudos devem reavaliar o grau de ameaça dessa espécie no Estado.

Myotis ruber apresenta distribuição restrita na América do Sul, ocorrendo no sul do Paraguai, leste da Argentina (Koopman 1982) e no Brasil nos estados de Minas Gerais, Pernambuco, Paraná, Rio de Janeiro, Rio Grande do Sul, Santa Catarina e São Paulo (Peracchi et al. 2006). É uma espécie considerada vulnerável pela lista de espécies ameaçadas de extinção do Brasil e do estado do Rio Grande do Sul, onde possui somente cinco registros. Foi capturada nos municípios de São Lourenço do Sul ( $31^{\circ} 18^{\prime}$ ' S e $\left.51^{\circ} 56^{\prime} \mathrm{W}\right)$ (Vieira 1955), Derrubadas $\left(27^{\circ} 10^{\prime} \mathrm{S}\right.$ e $\left.53^{\circ} 75^{\prime} \mathrm{W}\right)$ (Wallauer \& Albuquerque, 1986), São Francisco de Paula (29 $24^{\prime}$ S e $50^{\circ} 22^{\prime}$ W), Maquiné (293' S e $50^{\circ} 12^{\prime} \mathrm{W}$ ) e Barracão $\left(27^{\circ} 41^{\prime} \mathrm{S}\right.$ e $\left.51^{\circ} 26^{\prime \prime} \mathrm{W}\right)$ em áreas de Floresta
Estacional, Ombrófila Mista e Ombrófila Densa (Pacheco \& Freitas 2003). Sua distribuição atual no Estado está restrita aos limites norte, leste e sudeste, ocorrendo principalmente em Unidades de Conservação, em pontos isolados (Figura 1d). A ocorrência da espécie parece estar associada à vegetação de médio a grande porte, o que pode indicar maior sensibilidade às alterações ambientais se comparada a outras espécies de morcegos (Reis et al. 2003).

Foi examinada uma fêmea de $M$. ruber (ZUFSM 0404), capturada no dia 23/09/2005 às 19:50 horas (50 minutos após o pôr-dosol), com a utilização de redes-de-neblina, no município de Itaara $\left(29^{\circ} 32^{\prime} \mathrm{S}\right.$ e $\left.53^{\circ} 47^{\prime} \mathrm{W}\right)$ na área de abrangência da barragem Val de Serra, microbacia do rio Ibicuí Mirim, próxima à Reserva Biológica do Ibicuí-Mirim. O local de coleta apresenta vegetação arbustiva e 
se constitui em um ecótono floresta-campo. O indivíduo apresentava antebraço de 39,2 mm e massa de 6,6 g. Como essa espécie está na categoria vulnerável, segundo o Livro Vermelho da Fauna Ameaçada de Extinção no Rio Grande do Sul (Pacheco \& Freitas 2003), programas de conservação e manejo se fazem necessários para aumentar a proteção dessa e outras espécies na região.

As novas áreas de ocorrência dessas quatro espécies representam ampliações da distribuição e algumas ampliações para novas formações vegetais, especificamente para áreas de Floresta Estacional Decidual, na região central do Estado. Pode-se sugerir que algumas espécies como Histiotus montanus e Myotis ruber podem ser consideradas raras no Rio Grande do Sul, enquanto outras como Nyctinomops laticaudatus deveriam ter o seu grau de ameaça reavaliado, pois é uma espécie que aparenta ser abundante em áreas peri-urbanas de Santa Maria. Considerando-se os últimos registros de captura de $P$. bilabiatum, esta espécies aparenta ser relativamente bem distribuída no Estado do Rio Grande do Sul. Entretanto, poucos indivíduos são capturados em cada localidade, sugerindo que a espécie é pouco abundante localmente. Com isso, mostra-se que ainda se conhece muito pouco sobre a distribuição geográfica de morcegos no Rio Grande do Sul, sendo necessária a continuação dos estudos para se obter uma melhor avaliação sobre a ecologia, distribuição geográfica e status da conservação de morcegos neste Estado.

\section{Referências Bibliográficas}

BARQUEZ, R.M., GIANNINI, N.P. \& MARES, M.A. 1993. Guide of the bats of Argentina/ Guia de los murciélagos de Argentina. Oklahoma Museum of Natural History, Oklahoma.

CHEREM, J.J., SIMÕES-LOPES, P.C., ALTHOFF, S. \& GRAIPEL, M. E. 2004. Lista dos mamíferos do Estado de Santa Catarina, sul do Brasil. Mastozoología Neotropical 11(2):151-184.

FABIÁN, M.E., HARTZ, S.M. \& ARIGONY, T.H. A. 1990. Alimentação de Tadarida brasiliensis (Geoffroy, 1824) na região urbana de Porto Alegre, RS, Brasil (Chiroptera, Molossidae). Rev. Bras. Biol. 50:387-392.

FABIÁN, M.E., RUI, A.M. \& OLIVEIRA, K.P. 1999. Distribuição geográfica de morcegos Phyllostomidae (Mammalia: Chiroptera) no Rio Grande do Sul, Brasil. Iheringia, Sér. Zool. 87:143-156.

FABIÁN, M.E., GRILLO, H.C.Z. \& MARDER, E. 2006. Ocorrência de Histiotus montanus (Philippi \& Landbeck) (Chiroptera, Vespertilionidae) no Rio Grande do Sul, Brasil. Rev. Bras. Zool. 23(2):581-583.

GONZÁLEZ, J.C., FABIÁN, M.E. 1995. Uma nueva especie de murcielago para el estado de Rio Grande do Sul, Brasil: Myotis riparius Handley, 1960 (Chiroptera, Vespertilionidae). Comunicações do Museu Ciência e Tecnologia (PUCRS) 8:55-59.

KOOPMAN, K.F. 1982. Biogeography of the bats of South America. In Mammalian Biology in South America (M.A Mares \& H.H. Genoways, eds.). Special Publication Series, Pymatuning Laboratory of Ecology, University of Pittsburgh, Pittsburgh, p.273-302.

MARCHIORI, J. N. C. 2004. Fitogeografia do Rio Grande do Sul - Campos Sulinos. EST Edições, Porto Alegre.
MARINHO-FILHO, J. \& SAZIMA, I. 1998. Brazilian bats and conservation biology: a first survey. In Bat: biology and conservation (T.H. Kuntz \& P.A. Racey, eds.). Smithsonian Insitution Press, Washington, p.282-294.

MIRANDA, J.M.D., PULCHÉRIO-LEITE, A., MORO-RIOS, R.F. \& PASSOS, F.C. 2006. Primeiro registro de Histiotus montanus (Philippi \& Landbeck) para o Estado do Paraná, Brasil (Chiroptera, Vespertilionidae). Rev. Bras. Zool. 23(2):584-587.

PACHECO, S.M. \& FREITAS, T.R.O. 2003. Quirópteros. In Livro Vermelho da Fauna Ameaçada de Extinção no Rio Grande do Sul (C.S. Fontana, G.A. Bencke \& R.E. Reis, eds.). Edipucrs, Porto Alegre, p.493-497.

PACHECO, S.M. \& MARQUES, R.V. 2006. Conservação de morcegos no Rio Grande do Sul. In Mamíferos do Brasil: genética, sistemática, ecologia e conservação. (T.R.O. Freitas, E. Vieira, S.M. Pacheco \& A. Christoff, eds.). Suprema Gráfica Editora, São Carlos, p.91-106.

PERACCHI, A.L., LIMA, I.P., REIS, N.R., NOGUEIRA, M.R. \& FILHO, H.O. 2006. Ordem Chiroptera. In Mamíferos do Brasil (N.R. Reis, A.L. Peracchi, W.A. Pedro \& I.P. Lima, eds.). Universidade Estadual de Londrina, Londrina, p.153-230.

QUADROS, F.L.F \& PILLAR, V.P. 2002. Transições floresta-campo no Rio Grande do Sul. Ciência \& Ambiente 24:109-118.

REIS, N.R., BARBIERI, M.L.S., LIMA, I.P. \& PERACCHI, A. L. 2003. O que é melhor para manter a riqueza de espécies de morcegos (Mammalia, Chiroptera): um fragmento florestal grande ou vários fragmentos de pequeno tamanho? Rev. Bras. Zool. 20(2):225-230.

RUI, A.M., FABIÁN, M.E. \& MENEGHETI, J.O. 1999. Distribuição geográfica e análise morfológica de Artibeus lituratus Olfers e de Artibeus fimbriatus Gray (Chiroptera, Phyllostomidae) no Rio Grande do Sul, Brasil. Rev. Bras. Zool. 6(2):447-460.

SAZIMA, I., FISCHER, W.A., SAZIMA, M. \& FISCHER, E.A. 1994. The fruit bat Artibeus lituratus as a forest and city dweller. Ciência e Cultura 46(3):164-168.

SILVA, F. 1975. Três novas ocorrências de quirópteros para o Rio Grande do Sul, Brasil (Mammalia, Chiroptera). Iheringia, Sér. Zool. 46:51-53.

SILVA, F. \& SOUZA, M.F.B. 1980. Tadarida laticaudata Geoffroy 1805 , nova ocorrência para o Estado do Rio Grande do Sul, Brasil (Chiroptera, Mammalia). Iheringia, Sér. Zool. 56:3-5.

VIEIRA, C. 1955. Lista remissiva dos mamíferos do Brasil. Arquivos de Zoologia 8:341-474.

VOSS, W.A. 1973. Ensaio de lista sistemática dos mamíferos do Rio Grande do Sul, Brasil. Pesquisas 25:1-35.

WALLAUER, J.P. \& ALBUQUERQUE, E.P. 1986. Lista preliminar dos mamíferos observados no Parque Estadual do Turvo, Tenente Portela, Rio Grande do Sul, Brasil. Roessléria 8:179-185.

WALLAUER, J.P., BECKER, M., MARINS-SÁ, L.G., LIERMANN, L.M., PERRETTO, S.H. \& SCHERMACK, V. 2000. Levantamento dos mamíferos da Floresta Nacional de Três Barras - Santa Catarina. Biotemas 13(1):103-127.

WEBER, M.M., CÁCERES, N.C., LIMA, D.O., CAMILOTTI, V.L., ROMAN, C. \& NETO, L.T. 2006. Mammalia, Chiroptera, Phyllostomidae, Platyrrhinus lineatus: Range expansion to the state of Rio Grande do Sul, Brazil. Check List 2(3):96-98. 